\title{
ARTICLE \\ Overnight memory consolidation facilitates rather than interferes with new learning of similar materials - a study probing NMDA receptors
}

\author{
M. Alizadeh Asfestani ${ }^{1}$, E. Braganza ${ }^{1}$, J. Schwidetzky ${ }^{1}$, J. Santiago ${ }^{1,2,3}$, S. Soekadar ${ }^{4}$, J. Born ${ }^{1,5}$ and G. B. Feld $^{1,6,7}$
}

\begin{abstract}
Although sleep-dependent consolidation and its neurochemical underpinnings have been strongly researched, less is known about how consolidation during sleep affects subsequent learning. Since sleep enhances memory, it can be expected to pro-actively interfere with learning after sleep, in particular of similar materials. This pro-active interference should be enhanced by substances that benefit consolidation during sleep, such as D-cycloserine. We tested this hypothesis in two groups (Sleep, Wake) of young healthy participants receiving on one occasion D-cycloserine $(175 \mathrm{mg})$ and on another occasion placebo, according to a doubleblind balanced crossover design. Treatment was administered after participants had learned a set of word pairs (A-B list) and before nocturnal retention periods of sleep vs. wakefulness. After D-cycloserine blood plasma levels had dropped to negligible amounts, i.e., the next day in the evening, participants learned, in three sequential runs, new sets of word pairs. One list-to enhance interference-consisted of the same cue words as the original set paired with a new target word (A-C list) and the other of completely new cue words (D-E set). Unexpectedly, during post-retention learning the A-C interference list was generally better learned than the completely new D-E list, which suggests that consolidation of previously encoded similar material enhances memory integration rather than pro-active interference. Consistent with this view, new learning of word pairs was better after sleep than wakefulness. Similarly, D-cycloserine generally enhanced learning of new word pairs, compared to placebo. This effect being independent of sleep or wakefulness, leads us to speculate that D-cycloserine, in addition to enhancing sleep-dependent consolidation, might mediate a time-dependent process of active forgetting.
\end{abstract}

Neuropsychopharmacology (2018) 43:2292-2298; https://doi.org/10.1038/s41386-018-0139-0

\section{INTRODUCTION}

The relationship between sleep and memory maintenance has received detailed attention in the last 20 years [1,2] and there is widespread interest in enhancing this beneficial effect of sleep on memory [3], e.g., by enhancing neuronal oscillations [4, 5] or externally cueing replay $[6,7]$ i.e., processes that support sleepdependent memory. We recently demonstrated that the $\mathrm{N}$ methyl-D-aspartate (NMDA) receptor co-agonist D-cycloserine powerfully enhances sleep-dependent declarative memory consolidation when administered before sleep [8]. It is, however, completely unclear, how this enhancement affects the subtle balance of encoding and memory maintenance in the brain [9]. This is especially interesting as sleep has been suggested to also benefit new learning [10].

One of the first reports investigating the effect of sleep on memory was by Jenkins and Dallenbach [11], who famously hypothesized that sleep enhances memory not via an active process but by shielding it from interference, a line of argument that remains popular [12]. However, since this proposal it has been convincingly shown across species, modalities and paradigms that during sleep, memory is actively strengthened by the repeated replay of traces that were encoded during prior phases of wakefulness [6, 7, 13-15]. Intriguingly, it has also been shown that this sleep-dependent consolidations makes memory traces more robust towards retroactive interference [16], i.e., to the interfering influence of learning new information that deteriorates the original trace even if it had previously been successfully encoded [17]. When participants in this experiment learned a set of word pairs (A-B) before sleep and had to learn a retro-actively interfering set of word pairs $(A-C)$ after sleep (but before retrieval), the effect of sleep on memory retention was enhanced. Moreover, in a study where participants encoded while exposed to the smell of roses, re-exposing them to this odor cue during sleep made the associated memory robust to retro-active interference and the same treatment during wakefulness had the opposite effect [18]. These findings pose the intriguing question whether the reduction in memories' susceptibility to retro-active interference during sleep is due to a strengthening of the original trace that would be accompanied by enhanced pro-active interference, i.e., whether new memory traces are harder to establish if they overlap with these stronger old memory traces [17]

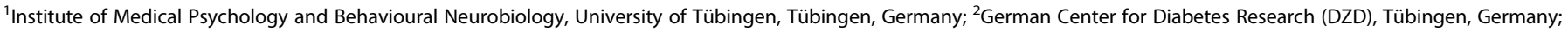

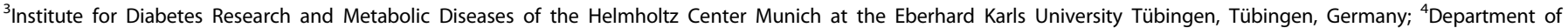

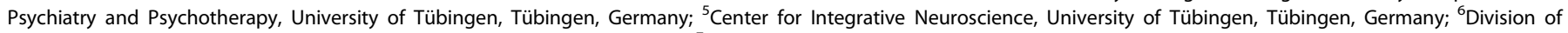

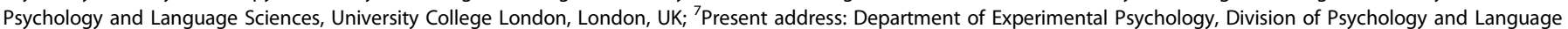
Science, Institute of Behavioural Neuroscience, University College London, 26 Bedford Way, London WC1H 0AP, UK Correspondence: M. Alizadeh Asfestani (marjan.alizadeh-asfestani@uni-tuebingen.de) or G. B. Feld (g.feld@ucl.ac.uk)
} 
The oscillatory properties of sleep that support the consolidation process [19] are ideally suited to drive the strengthening of memory traces via long-term potentiation (LTP) [20], which occurs mainly at glutamatergic synapses and is mediated by NMDA receptors [21, 22]. Accordingly, we administered D-cycloserine, a drug that supports NMDA receptor activation by binding to its glycine-binding site [23], to participants after they learned word pairs, so that peak plasma concentration occurred during the first half of the sleep phase [8]. Enhancing NMDA receptor activation benefited the sleep-dependent consolidation specifically of the word pairs if given during sleep and thus represents the ideal model to test whether memory traces enhanced by sleep introduce detrimental pro-active interference on new learning.

To test this we asked participants to learn a list of word pairs (A-B) and then enhanced sleep-dependent consolidation of these memories by administering D-cycloserine [8]. We expected that, when participants learned a new list of word pairs $(A-C)$ the next evening (i.e., after twice the drug half-life), performance would be reduced under treatment compared to placebo due to enhanced pro-active interference of the more strongly consolidated memory. To specify whether this effect depends on the item specificity of proactive interference, participants also learned new word pairs that did not overlap with the original list (D-E) and we expected that performance on this list would not be affected by treatment. We also tested a group of participants that did not sleep during the retention interval to test our hypothesis that these effects are mediated by processes active only during sleep.

\section{METHODS}

Participants

Fifty-one participants completed the study in the sleep $(n=30)$ and the Wake $(n=21)$ conditions. Participants were healthy, non-smoking, native German speaking men, age range between 18 and 30 years old, with a body mass index between 19 and 26 $\mathrm{kg} / \mathrm{m}^{2}$. This narrow range of inclusion criteria was chosen to test our hypothesis in a homogenous sample thereby limiting the amount of noise from variables of non-interest. Before starting the study a routine medical examination was performed for all the participants to exclude any psychiatric, neurological or endocrine diseases, participants who took regular medication were also excluded. The medical screening relied on a structured interview asking for current or past diagnosed conditions, a physical examination as well as a blood pressure and blood-screening test (white cells, red cells, hemoglobin, sodium, potassium, calcium, chloride, glucose, bilirubin, alanine transaminase, alkaline phosphatase, gamma-glutamyltransferase, C-reactive protein, prothrombin time, partial thromboplastin time) and only healthy participants were included. Participants were pre-screened via telephone making sure they did not take any acute medication at the time of the experiments and that they reported a normal sleep-wake cycle, no shift work, night work or intercontinental flights ( $>4 \mathrm{~h}$ time difference) for at least 6 weeks before the experiments. They were instructed to keep a steady sleep schedule in the week before the experiment (approximately sleeping from 23:00 to 07:00 each night), to go to bed at 23:00 the night before experiments, to get up at 07:00 on experimental days and, during these days, not to take any naps, no caffeinecontaining drinks after 13:00 and also not to consume alcohol starting one day before the experimental nights. Adherence to these rules was assessed with a questionnaire at the very beginning of each experimental condition and experiments were aborted and rescheduled, if gross deviation from this plan was found. This questionnaire also asked about acute medication and drug use as well as stressful events. Before the sleep experiment, participants took part in an adaptation night under conditions of

\section{A Study design}

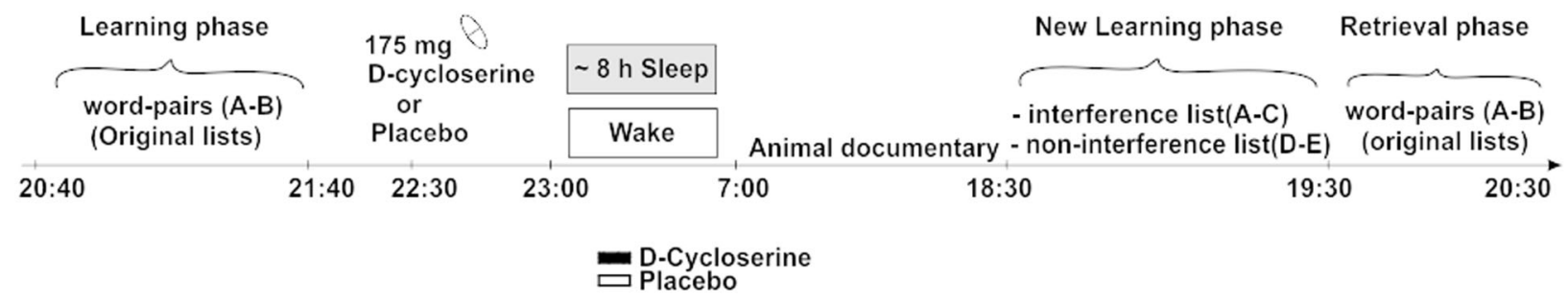
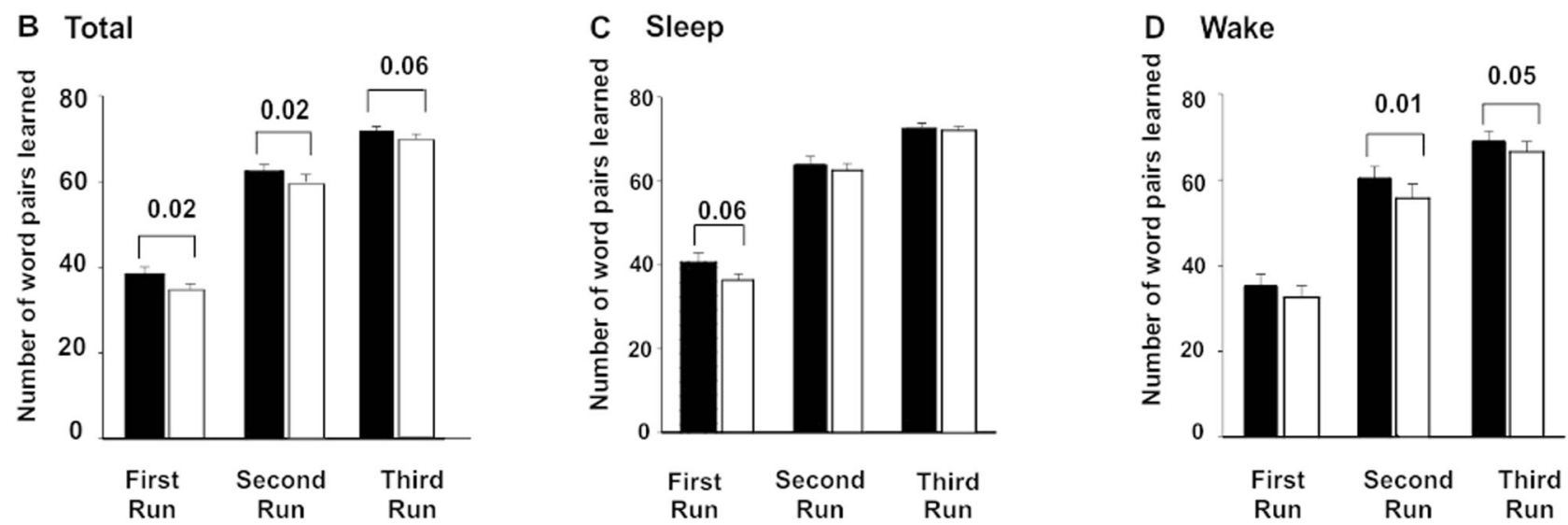

Fig. 1 a Participants first learned 80 word pairs (A-B) up to a criterion of $60 \%$, by a repeated cued recall procedure (see Methods section for details). Afterwards at $\sim 22: 30$, they took $175 \mathrm{mg}$ D-cycloserine or placebo. At 23:00 the participants in the Sleep group went to bed and polysomnographic recording was performed, whereas the Wake participants watched documentaries about planets. All participants received breakfast at 07:45 and watched animal documentaries until 18:00. Afterwards, at 18:30, the participants learned 80 new word pairs in three consecutive runs and finally retrieved the original 80 word pairs. b Mean and standard error of the mean (SEM) of the amount of correctly recalled word pairs in total, $\mathbf{c}$ in the Sleep experiment, and $\mathbf{d}$ in the Wake experiment during the three runs of the New Learning phase are shown 
the experiment, which included the placement of electrodes for polysomnographic recordings. The experiments were approved by the ethics committee of the University of Tübingen. We obtained written informed consent from all participants before their participation.

\section{Design and procedures}

The experiments followed a double-blind, placebo-controlled, within-subject crossover design, with Sleep vs. Wake betweensubjects groups. Within each group, participants took part in two identical experimental sessions with the exception of administration of D-cycloserine or placebo (D-cycloserine: Cycloserine Capsules ${ }^{\circledR}, 175 \mathrm{mg}$, the Chao Center for Industrial Pharmacy \& Contract Manufacturing, USA, plasma halftime: $10 \mathrm{~h}$, plasma maximum: 1-2 h; Fig. 1 summarizes study design). The dose of D-cycloserine was chosen to be the same as in our previous study [8], which effectively enhanced sleep-dependent consolidation of word pairs. D-cycloserine was applied at 22:30, i.e., 30 min before lights off in the Sleep group. This timing was chosen to maximize drug levels during the slow wave sleep rich first half of the night, which has been shown to be beneficial for the consolidation of word pairs. New learning of word pairs was scheduled the next evening, i.e., as late as possible without adding an additional night of sleep, in an attempt to minimize direct effects of the drug. The two experimental sessions were scheduled at least 14 days apart.

Participants arrived at 20:00 for each experimental night and first filled out questionnaires. In the Sleep group, polysomnography was prepared by applying electrodes. In the Wake group no polysomnography was assessed. Next, they learned the first set of declarative word pairs (original word pairs) between 20:40 and 21:40. Participants were informed that the word pairs would be recalled immediately and also the next evening, as well as, that they would learn new word pairs during the next evening. This was done to match expectations on the first session to those on the second session, where these essential procedures would be known by the participant. After learning they filled in questionnaires measuring mood and sleepiness and performed a reaction time task to measure vigilance (psychomotor vigilance task). At 22:30, Participants received the medication (D-cycloserine or placebo). At 23:00, the electrodes were connected to the amplifiers and lights were turned off, in the Sleep group. The Wake group watched astronomy documentaries while sitting in a comfy chair (two counterbalanced lists of films, one for each session) in the lab during this time and were monitored by the investigator through cameras at all times, who prevented them falling asleep unintentionally. Approximately every $1.5 \mathrm{~h}$, participants took a short walk together with the investigator to enhance wakefulness. After $\sim 8 \mathrm{~h}$ (between 06:45 and 07:15), the Sleep group was woken up (if possible from sleep stage 1 or 2). All participants first answered questionnaires measuring their mood and sleepiness and, afterwards, received a standardized breakfast and the Sleep group was allowed to shower to clean the electrode gel off their head. During the day, participants followed a tight protocol watching animal documentaries (two counterbalanced lists of films, one for each session) for $\sim 1 \frac{1}{2} \mathrm{~h}$ at a time (two episodes), where the investigator checked in on them every 15 min to ensure wakefulness. Each of these episodes was followed by a break to take a walk around the campus and participants received two snacks in the afternoons. This was done for $10 \mathrm{~h}$ only interrupted by lunch at the local canteen together with the investigator. This protocol was chosen to standardize the experience of the participants after drug application and to minimize the opportunity of newly encoding written words. This strategy included that participants were not allowed to use their mobile devices and the internet or read books and magazines during the experiment. Please note, however, that we cannot completely exclude that participants encoded new words. We chose not to completely deprive the participants from all sensory input, which would have minimized these opportunities even more, as it would have been nearly impossible to keep them awake at the same time. At 18:30, participants learned new word pairs and immediately recalled them. Next they recalled original word pairs they learned the day before. Finally, we again measured mood, sleepiness, and vigilance, as well as, word generation.

\section{Word-pair tasks}

Consolidation was measured using 80 slightly associated word pairs (A-B list, e.g., Democracy-System) in two lists (original lists). The word pairs were presented on a computer screen for $4 \mathrm{~s}$ each with a $1 \mathrm{~s}$ inter-stimulus interval (ISI). After presenting both lists, the participant's memory was tested in a cued recall procedure by presenting only the first word and asking the participant to produce the associated word. This was done for each list individually. If the participant did not reach the criterion of $60 \%$ correct responses on one (or both) of the lists, only this list (or both lists) was presented again completely ( $3 \mathrm{~s}$ per pair) and cued recall was repeated. This was done until he reached the criterion. The amount of word pairs recalled during the last cued recall was used as measure of learning performance. The cued recall procedure as described above was performed again at the very end of the Retrieval phase (after the New Learning phase-see below). This was done, so that the participants formed the intention to retain the word pairs across each of the two sessions, which may have been undercut, if delayed retrieval did not take place in the first session. The intention to retain word pairs has previously been shown to be an essential factor driving sleepdependent memory consolidation [24]. Data from this retrieval were not analyzed as they are confounded by the prior new learning.

During the next evening, participants were presented a new set of 80 word pairs in two separate lists. One list with 40 word pairs (A-C list, Democracy-Equality) interfered with the original list, i.e., they contained a new sec word (C) paired with a cue word (A) of the original list (interference list), the other also consisting of 40 word pairs (D-E list, e.g., Painter-Pianist) was completely new (nointerference list). These two word-pair lists were also learned back to back in a balanced order and each pair was shown for $4 \mathrm{~s}(1 \mathrm{~s}$ ISI). The cued recall procedure described above was performed three times (run 1-run 3) and after runs 1 and 2 the word pairs were shown again for $3 \mathrm{~s}$ each. Note that we constructed six pairs of $A-B$ and corresponding A-C lists with 40 word pairs each. We counterbalanced which of the lists was used for the interference (two pairs per participant, $A-B$ and corresponding $A-C$ version) and non-interference conditions (four lists per participant, i.e., only the $A-B$ or the $A-C$ version of the remaining four pairs, referred to as $\mathrm{D}-\mathrm{E}$ lists below to convey that they were non-overlapping).

Polysomnography, sleep analysis, and EEG power analysis The EEG was recorded continuously from electrodes $(\mathrm{Ag}-\mathrm{AgCl})$ placed according to the 10-20 System, referenced to two linked electrodes attached to the mastoids. EEG signals were filtered between 0.03 and $35 \mathrm{~Hz}$, and sampled at a rate of $250 \mathrm{~Hz}$ using a Brain Amp DC (BrainProducts $\mathrm{GmbH}$, Munich, Germany). Additionally, horizontal and vertical eye movements (HEOG, VEOG) and the EMG (via electrodes attached to the chin) were recorded for standard polysomnography. Sleep architecture was determined according to standard polysomnographic criteria using EEG recordings from C3 and C4 [25]. Scoring was performed by an experienced technician who was blind to the assigned treatment (an additional expert was consulted for ambiguous epochs). For each night, total sleep time (TST), i.e., the time between the first detection of transition from sleep stage 1 to 2 and lights on, and time spent in the different sleep stages, i.e., wake; sleep stages 1, $2,3,4$; SWS (defined by the sum of sleep stage 3 and 4) and rapid eye movement (REM) sleep was calculated in minutes. 
Table 1. Mean (SEM) correctly recalled word pairs in the New Learning phase for the interference and no-interference conditions

\begin{tabular}{|c|c|c|c|c|c|c|c|}
\hline & \multicolumn{3}{|l|}{ Sleep } & \multicolumn{4}{|l|}{ Wake } \\
\hline & $\begin{array}{l}\text { D- } \\
\text { cycloserine }\end{array}$ & \multicolumn{2}{|c|}{ Placebo } & \multicolumn{2}{|c|}{$\begin{array}{l}\text { D- } \\
\text { cycloserine }\end{array}$} & \multicolumn{2}{|c|}{ Placebo } \\
\hline \multicolumn{8}{|l|}{ Interference } \\
\hline First run & $21.42(1.12)$ & 19.00 & $(0.93)$ & 19.38 & $(1.32)$ & 16.67 & (1.49) \\
\hline Second run & $31.85 \quad(0.82)$ & 31.27 & $(1.00)$ & 30.76 & (1.25) & 27.57 & (1.57) \\
\hline Third run & $36.08 \quad(0.53)$ & 36.31 & $(0.59)$ & 35.10 & $(1.13)$ & 33.00 & $(1.22)$ \\
\hline \multicolumn{8}{|c|}{ No-interference } \\
\hline First run & $19.27(1.28)$ & 17.38 & $(0.98)$ & 15.95 & $(1.61)$ & 16.00 & (1.62) \\
\hline Second run & $32.23 \quad(1.20)$ & 31.31 & $(0.99)$ & 29.67 & (1.62) & 28.19 & (1.97) \\
\hline Third run & $36.73 \quad(0.62)$ & 35.81 & $(0.65)$ & 34.00 & (1.34) & 33.57 & (1.26) \\
\hline
\end{tabular}

Control measures-vigilance, sleepiness, and mood ratings and test of encoding

Participant's sleepiness and mood was assessed using self-report measures. The Stanford Sleepiness Scale (SSS) [26] measures subjective sleepiness with one item and eight answer options ranging from one $=$ "Feeling active, vital, alert, or wide awake" to eight = "Asleep" (provided as an anchor). We assessed the participant's mood using the multidimensional mood questionnaire at three time points per session [27]. This questionnaire produces the three scales positive mood (high is positive), tiredness (low is tired), and calmness (high is calm). Objective vigilance was additionally tested using the psychomotor vigilance task (PVT; [28]. This 5-min version of the PVT required pressing a button as fast as possible whenever a bright millisecond clock presented on a dark computer screen started counting upward. After the button press, this clock displayed the reaction time. General capabilities of long-term memory retrieval were tested using a word generation task, which, e.g., has been used to diagnose such long-term memory capabilities in very mild dementia [29], but also requires executive function that is affected by total sleep deprivation [30]. Participants had to produce as many words as possible starting with a certain letter ( $P$ or $M)$ or belonging to a defined category (hobby or profession) during a time of 2 min each (Regensburger Wortflüssigkeitstest [WFT]; [31]). At the end of each session all participants were asked if they believed to have received the active agent or placebo.

Data reduction and statistical analysis

In the Sleep group two participants were excluded because of their extremely low learning performance (below seven word pairs in more than one list). After checking the sleep scoring, two participants were excluded because of disrupted sleep. Statistical analyses generally relied on analyses of variance (GLM; SPSS version 21.0.0 for Windows) including the repeated-measures factors Substance (D-cycloserine vs. placebo), Interference (interference vs. no-interference) and, where appropriate, the factor Runs $(1,2,3)$ pertaining to the three recalls during the New Learning phase, as well as, the between-subjects factor Sleep/ Wake. Greenhouse-Geisser correction of degrees of freedom was applied where necessary. Significant interactions were followed up by lower-level ANOVAs and post hoc $t$ tests.

\section{RESULTS}

Word pairs

Concerning the New Learning phase of word pairs on the second evening of each session, the interference list was learned significantly better than the no-interference list, with this effect being predominant on the first run (Interference $\times$ Run: $F_{(2,90)}=$ 9.035, $p=0.001$, First run: $\mathrm{F}_{(1,45)}=9.492, p=0.004$; Table 1). Trivially, performance improved across the three runs $\left(\mathrm{F}_{(2,90)}=\right.$ $832.695, p \leq 0.001)$. We also found a trend towards the Sleep group learning more new word pairs than the Wake group $\left(F_{(1,45)}=3.447, p=0.070\right.$, for main effect of the Sleep/Wake factor, i.e., not different between $A-C$ and $D-E$ lists). D-Cycloserine distinctly enhanced new learning of word pairs $20 \mathrm{~h}$ after administration $\left(F_{(1,45)}=6.512, p=0.014\right.$, for main effect of Substance). On average participants learned 2.7 more new word pairs in the $D$-cycloserine condition than in the Placebo condition (D-cycloserine: $57.30 \pm 1.40$, Placebo: $54.63 \pm 1.43$ ).

We also identified a three-way interaction of Substance $\times$ Run $\times$ Sleep/Wake $\left(F_{(2,90)}=3.514, p=0.034\right)$. This effect was mainly driven by word pairs being learned more in the Sleep group after D-cycloserine during the first run $\left(t_{(25)}=1.964, p=0.061\right)$, whereas word pairs being learned more in the Wake group after $D$ cycloserine during the second $\left(t_{(20)}=2.880, p=0.009\right)$ and third $\left(t_{(20)}=2.102, p=0.048\right)$ runs. All the other effects and interactions were non-significant (All $p \geq 0.114$; Table 1 ).

\section{Sleep stages}

Under D-cycloserine participants spent significantly more time (in minutes) in wakefulness and sleep stage 1 (Wake: $t_{(25)}=-2.737 p$ $=0.011$; stage $1: t_{(25)}=-2.661 p=0.013$, descriptive statistics are provided in Fig. 2) and less time in REM sleep $\left(t_{(25)}=2.768\right.$, $p=0.010)$. We also found a trend towards reduced time in sleep stage 2 in the $D$-cycloserine condition in comparison to placebo $\left(t_{(25)}=1.795, p=0.085\right)$, but there was no significant difference between the treatments in time spent in the other sleep stages (all $t \geq-0.356, p \geq 0.451)$ or total sleep time $\left(t_{(25)}=-0.509, p=0.615\right)$. Time spent in sleep stage 4 was positively correlated to performance during Run 1 and Run 3 of the New Learning phase for the no interference list in the placebo condition $(r=0.34$, $p=0.091$ and $r=0.42, p=0.032$, respectively). Conversely, time spent awake was negatively related to performance during Run 3 of the New Learning phase for the no interference list in the placebo condition $(p=0.017, r=0.47)$. Of note, the reported correlation is not evident, if sleep stages 3 and 4 are combined to SWS (all $p \geq 0.23$ ).

\section{Control measures}

As can be expected, in the Wake group, sleep deprivation led to increased subjective sleepiness (measured by Stanford Sleepiness Scale) in the morning and at retrieval $\left(\mathrm{F}_{(1,45)}=41.07, p \leq 0.001\right.$ and $\mathrm{F}_{(1,45)}=52.74, \quad p \leq 0.001$, respectively), as well as, reduced objective vigilance (measured by PVT) at retrieval $\left(F_{(1,45)}=4.03\right.$, $p=0.051$ ) compared to the Sleep group. However, in both groups

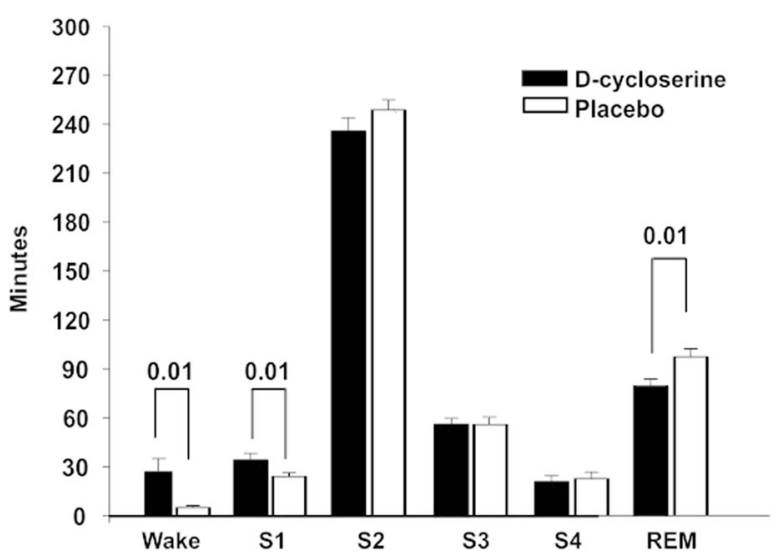

Fig. 2 Mean (SEM) time spent in the different sleep stages Wake, S1 (sleep stage 1), S2 (sleep stage 2), S3 (sleep stage 3), S4 (sleep stage 4 ), and REM (rapid eye movement sleep) sleep in minutes 
Table 2. Means (SEM) of performance are given for the control measures

\begin{tabular}{llll} 
Sleep & & Wake & \\
\cline { 1 - 1 } $\begin{array}{l}\text { D- } \\
\text { cycloserine }\end{array}$ & Placebo & $\begin{array}{l}\text { D- } \\
\text { cycloserine }\end{array}$ & Placebo \\
\hline
\end{tabular}

Stanford Sleepiness Scale

\begin{tabular}{|c|c|c|c|c|c|c|c|c|}
\hline Learning & 3.23 & $(0.17)$ & 3.77 & $(0.21)$ & 2.81 & $(0.25)$ & 2.95 & $(0.26)$ \\
\hline Morning & 3.65 & $(0.26)$ & 3.42 & $(0.17)$ & 5.05 & $(0.23)$ & 5.38 & $(0.22)$ \\
\hline Retrieval & 2.81 & $(0.24)$ & 2.92 & $(0.23)$ & 5.43 & $(0.28)$ & 5.14 & $(0.29)$ \\
\hline \multicolumn{9}{|c|}{ Psychomotor vigilance task } \\
\hline Learning & 3.74 & $(0.07)$ & 3.70 & $(0.07)$ & 3.73 & $(0.09)$ & 3.74 & $(0.09)$ \\
\hline Retrieval & 3.82 & $(0.08)$ & 3.78 & $(0.07)$ & 3.60 & $(0.09)$ & 3.55 & $(0.08)$ \\
\hline \multicolumn{9}{|c|}{ Multidimensional mood questionnaire } \\
\hline \multicolumn{9}{|c|}{ Learning } \\
\hline $\begin{array}{l}\text { Positive } \\
\text { mood }\end{array}$ & 16.88 & $(0.51)$ & 16.46 & $(0.66)$ & 17.29 & $(0.44)$ & 17.05 & $(0.41)$ \\
\hline Tiredness & 12.19 & $(0.47)$ & 11.07 & $(0.58)$ & 13.90 & $(0.64)$ & 13.14 & $(0.60)$ \\
\hline Calmness & 16.69 & $(0.38)$ & 15.85 & $(0.68)$ & 16.00 & $(0.56)$ & 15.57 & $(0.62)$ \\
\hline \multicolumn{9}{|l|}{ Morning } \\
\hline $\begin{array}{l}\text { Positive } \\
\text { mood }\end{array}$ & 16.08 & $(0.66)$ & 17.00 & $(0.50)$ & 13.19 & $(0.65)$ & 11.76 & $(0.73)$ \\
\hline Tiredness & 12.46 & $(0.77)$ & 14.08 & $(0.59)$ & 8.19 & $(0.74)$ & 6.90 & (0.64) \\
\hline Calmness & 15.96 & $(0.55)$ & 16.62 & $(0.52)$ & 13.19 & $(0.68)$ & 12.67 & $(0.70)$ \\
\hline \multicolumn{9}{|l|}{ Retrieval } \\
\hline $\begin{array}{l}\text { Positive } \\
\text { mood }\end{array}$ & 16.15 & $(0.84)$ & 15.92 & $(0.76)$ & 13.48 & $(0.75)$ & 13.24 & $(0.70)$ \\
\hline Tiredness & 12.85 & $(0.81)$ & 13.08 & $(0.70)$ & 7.00 & (0.69) & 6.67 & (0.64) \\
\hline Calmness & 14.27 & $(0.80)$ & 14.46 & $(0.74)$ & 12.00 & $(0.75)$ & 12.24 & $(0.69)$ \\
\hline \multicolumn{9}{|c|}{ Word fluency task } \\
\hline Category & 19.35 & $(1.27)$ & 18.58 & $(0.95)$ & 16.71 & $(0.98)$ & 17.24 & $(0.98)$ \\
\hline Letter & 17.96 & $(0.85)$ & 18.35 & $(1.01)$ & 15.14 & (1.14) & 15.67 & (0.79) \\
\hline
\end{tabular}

Subjective sleepiness (Stanford Sleepiness Scale), objective vigilance (Psychomotor Vigilance Task), mood (Multidimensional mood questionnaire), and general retrieval performance (Word Fluency Test). Learning (after the original Learning phase), Morning (at 07:15), Retrieval (after the Retrieval phase).

we found no differences between the treatments in the psychomotor vigilance task (PVT, all $p \geq 0.196$ ). In the Sleep group, subjective "tiredness" in the morning after nocturnal sleep, was enhanced in the D-cycloserine group $\left(t_{(25)}=-2.534, p=0.018\right.$; Table 2). In the Wake group, subjects in the morning after Dcycloserine showed trend-wise higher "good mood" and less "tiredness" ("tiredness": $t_{(20)}=1.910, p=0.071$, "good mood": $t_{(20)}$ $=1.805, p=0.086$; Table 2) than after placebo. Sleepiness (on the SSS did not differ between substance conditions at all times (all $p \geq 0.167$ ). Also, we did not have any significant differences between substance conditions in the general retrieval performance as measured by the word generation task within any of the groups (all $p \geq 0.503$ ), however, the Sleep group produced more words compared to the Wake group in an overall analysis $\left(\mathrm{F}_{(1,45)}=\right.$ 5.02, $p=0.030$ ). Participants in both groups were not able to discriminate between $\mathrm{D}$-cycloserine and placebo (McNemars' exact test: $p \geq 0.774$ ).

\section{DISCUSSION}

We expected that learning of new word pairs will be decreased after sleep under D-cycloserine in comparison to placebo, due to enhanced pro-active interference by the consolidated memory. This effect was predicted to be facilitated, if the new word pairs shared the consolidated word-pair's cue word. In contrast, we found that sharing a cue word with the original list enhanced new learning rather than impaired it. Also, our results provide evidence that new learning was generally facilitated by sleep and by $D^{-}$ cycloserine. Notably the enhancing effect of D-cycloserine was independent of whether it was given before sleep or wakefulness, which was also unexpected. The effects of D-cycloserine on sleep architecture replicated earlier findings of increased wake and light sleep while REM sleep was reduced [8], suggesting a robust albeit mild alerting effect of the drug. Generally, these findings suggest that pro-active interference that is predominant immediately after learning does not carry over to longer retention intervals but rather is reversed by consolidation to aid new learning. Accordingly, enhancing sleep-dependent consolidation of memory traces appears to pro-actively support new learning. Moreover, the effect of D-cycloserine being independent of sleep and associated consolidation, suggests additional time-dependent mechanisms supporting new learning perhaps by inducing active forgetting.

Our finding of better encoding in the interference condition than in the no-interference condition indicates that rather than producing pro-active interference and thereby impairing new learning our interference condition enhanced new learning. This cannot be explained by the initial memory merely decaying across time and thereby reducing its pro-active influence, as this would not enhance performance on the interference above and beyond the no-interference condition. It has been proposed that new information can be learned more easily, if it can be integrated with existing knowledge [32]. Theoretically consolidation during sleep may derive such knowledge by abstracting from episodes [33]. However, empiric evidence suggests that knowledge may be built in a time-dependent rather than a sleep-dependent manner [34], which is consistent with our data revealing that improved learning of interfering materials is independent of prior sleep or wakefulness. Intriguingly, work in rodents suggests, that after a schema is established, new learning is facilitated [35] and subsequent work has shown that such schema-dependent encoding may access the cortical store directly [36]. Alternatively, the present effect may rely on map-like representations of concepts established within the entorhinal grid code [37], which has been shown to encode complex associative memories [38]. Essentially, this question needs to be addressed by additional experiments that go beyond the scope of the current study and establish when pro-active interference is overridden by knowledge abstraction.

The trend-wise enhanced encoding in the Sleep versus the Wake group, corresponds to earlier findings of enhanced encoding after sleep [10], however, this effect may also have been due to sleep deprivation leading to reduced performance in the Wake group. The absence of a more pronounced difference in encoding performance between the sleep and the wake group could also be indicative of a ceiling effect, which would be most pronounced in the third run of the New Learning phase. In support of an active contribution of sleep to improved encoding, we found that time spent in sleep stage 4 was positively related to New Learning phase performance in the placebo condition. While this is in line with causal experiments that showed that boosting slow wave activity can enhance the encoding of declarative memories after sleep [39], an important function may also be performed by sleep spindles during sleep stage 2 [10]. In contrast to findings that suggest REM sleep down-scales synaptic weights in the hippocampus [40], we found improved encoding after Dcycloserine combined with the drug's substantial suppression of REM. Interestingly, we found that there was a significant three-way interaction (between Substance, Sleep/Wake, and Runs), which appeared to mainly reflect that in the Sleep group D-cycloserineenhanced new learning on run 1, whereas in the Wake group the NMDA receptor co-agonist-enhanced learning on runs 2 and 3. 
This can be interpreted as the Sleep group already reaching ceiling levels after run 1, because of the additional boost in learning through sleep. The Wake group on the other hand had more opportunity to increase learning later on in the task. In essence, we suggest that this interaction effect is mediated by sleep-dependent increases in new learning that are independent of the increases in new learning induced by D-cyloserine.

Thus, unexpectedly, D-cycloserine not only enhanced new learning when administered before sleep but also when administered before a wake retention period. This is difficult to integrate. We suspect that this effect might reflect an involvement of NMDA receptor activation in sleep-independent processes that renormalize synaptic weights and generally free capacity for novel encoding (see refs. [41, 42] for opposing remarks) that have recently been shown to also occur during wakefulness [43]. Active decay is a form of renormalization that has been suggested to occur at glutamatergic synapses [44]. Studies of object-location and associative memories in rats have shown that such decay can be prevented by blocking the removal of a-amino-3-hydroxy-5methyl-4-isoxazolepropionic acid (AMPA) receptors from the synapse [45]. Similarly, blocking NMDA receptors for prolonged periods impaired forgetting of spatial memory in rats [46]. As activating the NMDA receptor in specific ways induces AMPAreceptor endocytosis [47], it is tempting to speculate that the present finding of a generally enhanced encoding (after wake as well as sleep retention periods) involves D-cycloserine sensitizing NMDA receptors to ambient glutamate levels [48], which drives forgetting and frees up capacity for new learning. It is important to note that although we aimed to minimize direct effects of Dcycloserine on encoding by timing New Learning as late as possible, i.e., after two times the half-life of the drug, the drug has also been shown to directly enhance performance when administered before learning [49], and a residual direct influence on new learning cannot be ruled out completely. Remarkably, Dcycloserine's effect on memory has been suggested to rely on lower-level, automatic mechanisms rather than directly affecting high-level processes such as declarative learning [50], which nicely aligns with drug effects in the present study and our previous report [8] affecting processing not under immediate explicit control, i.e., forgetting and consolidation, respectively.

Since sleep-dependent memory consolidation might be influenced by sex and sex hormones [51] the present study is limited, inasmuch as, only men were investigated, which was done to limit risks of the drug to unborn life and reduce noise by assessing a homogenous sample. Also, we only considered a high-level declarative learning task in this study and sleep has been shown also to re-process, e.g., motor sequence memories [52]. However, no effect of sleep on subsequent encoding was found for this task in a nap study [10]. Similarly, we did not manipulate the difficulty of our memory task, which has been shown to modulate the effects of sleep-dependent memory consolidation [16, 53], and thus may have influenced the degree of sleep related interference produced by our lists.

In conclusion, we found that overnight retention periods after learning facilitated new learning in particular of interfering materials. Sleep as well as D-cycloserine generally enhanced new learning, and these effects might partly originate from their consolidating influence of the originally learned A-B word pairs, that might facilitate transfer learning of new wordlists (including A-C and D-E lists). The effect of D-cycloserine likewise observed after wake periods also suggests a contribution of NMDA receptormediated active decay [44] that is established as a form of sleepindependent synaptic renormalization $[42,43]$. Examining how this form of forgetting interacts with sleep-dependent forms of synaptic renormalization [41] and sleep-dependent memory consolidation [2], will be of essence to understand how consolidation and forgetting sustain long-term memory and new learning [54].

\section{ACKNOWLEDGEMENTS}

We gratefully acknowledge Marion Inostroza for supporting all phases of the project and also would like to thank Michael Radloff for assisting data collection. This research was supported by a grant from the Deutsche Forschungsgemeinschaft (DFG) SFB 654 "Plasticity and Sleep" and by grants from the German Federal Ministry of Education and Research (BMBF) to the German Center for Diabetes Research (DZD e.V.; 01GI0925). G.B.F. is currently receiving a personal stipend from the DFG to conduct research at the University College London (FE 1617/1-1).

\section{ADDITIONAL INFORMATION}

Competing interests: The authors declare no competing interests.

Publisher's note: Springer Nature remains neutral with regard to jurisdictional claims in published maps and institutional affiliations.

\section{REFERENCES}

1. Rasch B, Born J. About sleep's role in memory. Physiol Rev. 2013;93:681-766. https://doi.org/10.1152/physrev.00032.2012

2. Diekelmann S, Born J. The memory function of sleep. Nat Rev Neurosci. 2010;11:114-26. https://doi.org/10.1038/nrn2762

3. Feld GB, Diekelmann S. Sleep smart-optimizing sleep for declarative learning and memory. Front Psychol. 2015;6:622 https://doi.org/10.3389/fpsyg.2015.00622

4. Marshall L, Molle M, Hallschmid M, Born J. Transcranial direct current stimulation during sleep improves declarative memory. J Neurosci. 2004;24:9985-92. https:// doi.org/10.1523/JNEUROSCI.2725-04.2004

5. Ngo HV, Martinetz T, Born J, Molle M. Auditory closed-loop stimulation of the sleep slow oscillation enhances memory. Neuron. 2013;78:545-53. https://doi. org/10.1016/j.neuron.2013.03.006

6. Rasch B, Buchel C, Gais S, Born J. Odor cues during slow-wave sleep prompt declarative memory consolidation. Science. 2007;315:1426-9. https://doi.org/ 10.1126/science.1138581

7. Rudoy JD, Voss JL, Westerberg CE, Paller KA. Strengthening individual memories by reactivating them during sleep. Science. 2009;326:1079 https://doi.org/ 10.1126/science. 1179013

8. Feld GB, Lange T, Gais S, Born J. Sleep-dependent declarative memory consolidation-unaffected after blocking NMDA or AMPA receptors but enhanced by NMDA coagonist D-cycloserine. Neuropsychopharmacology. 2013;38:2688-97. https://doi.org/10.1038/npp.2013.179

9. Richards BA, Frankland PW. The persistence and transience of memory. Neuron. 2017;94:1071-84. https://doi.org/10.1016/j.neuron.2017.04.037

10. Mander BA, Santhanam S, Saletin JM, Walker MP. Wake deterioration and sleep restoration of human learning. Curr Biol. 2011;21:R183-4. https://doi.org/10.1016/ j.cub.2011.01.019

11. Jenkins JG, Dallenbach KM. Obliviscence during sleep and waking. Am J Psychol. 1924;35:605-12.

12. Mednick SC, Cai DJ, Shuman T, Anagnostaras S, Wixted JT. An opportunistic theory of cellular and systems consolidation. Trends Neurosci. 2011;34:504-14. https://doi.org/10.1016/j.tins.2011.06.003

13. Sadowski JH, Jones MW, Mellor JR. Sharp-wave ripples orchestrate the induction of synaptic plasticity during reactivation of place cell firing patterns in the hippocampus. Cell Rep. 2016;14:1916-29. https://doi.org/10.1016/j.celrep.2016. 01.061

14. van de Ven GM, Trouche S, McNamara CG, Allen K, Dupret D. Hippocampal offline reactivation consolidates recently formed cell assembly patterns during sharp waveripples. Neuron. 2016;92:968-74. https://doi.org/10.1016/j.neuron.2016.10.020

15. Wilson MA, McNaughton BL. Reactivation of hippocampal ensemble memories during sleep. Science. 1994;265:676-9.

16. Ellenbogen JM, Hulbert JC, Stickgold R, Dinges DF, Thompson-Schill SL. Interfering with theories of sleep and memory: sleep, declarative memory, and associative interference. Curr Biol. 2006;16:1290-4. https://doi.org/10.1016/j. cub.2006.05.024

17. Osgood CE. An investigation into the causes of retroactive interference. J Exp Psychol. 1948;38:132-54.

18. Diekelmann S, Wilhelm I, Wagner U, Born J. Elevated cortisol at retrieval suppresses false memories in parallel with correct memories. J Cogn Neurosci. 2011;23:772-81. https://doi.org/10.1162/jocn.2010.21493

19. Staresina BP, Bergmann TO, Bonnefond $M$, van der Meij $R$, Jensen O, Deuker $L$ et al. Hierarchical nesting of slow oscillations,spindles and ripples in the human hippocampus during sleep. Nat Neurosci. 2015;18:1679-86. https://doi.org/ 10.1038/nn.4119

20. King C, Henze DA, Leinekugel X, Buzsaki G. Hebbian modification of a hippocampal population pattern in the rat. J Physiol. 1999;521:159-67. 
21. Malenka RC, Bear MF. LTP and LTD: an embarrassment of riches. Neuron. 2004:44:5-21. https://doi.org/10.1016/j.neuron.2004.09.012

22. Malenka RC, Nicoll RA. Long-term potentiation-a decade of progress? Science. 1999;285:1870-4.

23. Sheinin A, Shavit S, Benveniste M. Subunit specificity and mechanism of action of NMDA partial agonist D-cycloserine. Neuropharmacology. 2001;41:151-8.

24. Wilhelm I, Diekelmann S, Molzow I, Ayoub A, Molle M, Born J, et al. Sleep selectively enhances memory expected to be of future relevance. J Neurosci. 2011;31:1563-9. https://doi.org/10.1523/JNEUROSCI.3575-10.2011

25. Rechtschaffen A, Kales A. A manual of standardized terminology, techniques and scoring system for sleep stages of human subjects. Bethesda, MD: US Department of Health, Education and Welfare (1968). https://www.ncbi.nlm.nih.gov/ nlmcatalog/?term =Rechtschaffen\%20A,\%20Kales\%20A\%20\%281968\%29

26. Hoddes E, Zarcone V, Smythe H, Phillips R, Dement WC. Quantification of sleepiness: a new approach. Psychophysiology. 1973;10:431-6.

27. Hinz A, Daig I, Petrowski K, Brahler E. [Mood in the German population: norms of the Multidimensional Mood Questionnaire MDBF]. Psychother Psychosom Med Psychol. 2012;62:52-7. https://doi.org/10.1055/s-0031-1297960

28. Dinges DF, Pack F, Williams K, Gillen KA, Powell JW, Ott GE, et al. Cumulative sleepiness, mood disturbance, and psychomotor vigilance performance decrements during a week of sleep restricted to 4-5 h per night. Sleep. 1997;20:267-77.

29. Gomez RG, White DA. Using verbal fluency to detect very mild dementia of the Alzheimer type. Arch Clin Neuropsych. 2006;21:771-5. https://doi.org/10.1016/j. acn.2006.06.012

30. Jones K, Harrison Y. Frontal lobe function, sleep loss and fragmented sleep. Sleep Med Rev. 2001;5:463-75.

31. Aschenbrenner A, Tucha O, Lange K. RWT Regensburger Wortflüssigkeits-Test. Handanweisung. Göttingen: Hogrefe Verlag; 2000.

32. van Kesteren MT, Rijpkema M, Ruiter DJ, Morris RG, Fernandez G. Building on prior knowledge: schema-dependent encoding processes relate to academic performance. J Cogn Neurosci. 2014;26:2250-61. https://doi.org/10.1162/jocn_a_00630

33. Lewis PA, Durrant SJ. Overlapping memory replay during sleep builds cognitive schemata. Trends Cogn Sci. 2011;15:343-51. https://doi.org/10.1016/j.tics.2011. 06.004

34. Hennies N, Lewis PA, Durrant SJ, Cousins JN, Ralph MA. Time- but not sleepdependent consolidation promotes the emergence of cross-modal conceptual representations. Neuropsychologia. 2014;63:116-23. https://doi.org/10.1016/j. neuropsychologia.2014.08.021

35. Tse D, Langston RF, Kakeyama M, Bethus I, Spooner PA, Wood ER, et al. Schemas and memory consolidation. Science. 2007;316:76-82. https://doi.org/10.1126/ science. 1135935

36. Tse $D$, Takeuchi $T$, Kakeyama $M$, Kajii $Y$, Okuno $H$, Tohyama $C$, et al. Schemadependent gene activation and memory encoding in neocortex. Science. 2011;333:891-5. https://doi.org/10.1126/science.1205274

37. Constantinescu AO, O'Reilly JX, Behrens TEJ. Organizing conceptual knowledge in humans with a gridlike code. Science. 2016;352:1464-8. https://doi.org/10.1126/ science.aaf0941

38. Garvert MM, Dolan RJ, Behrens TE. A map of abstract relational knowledge in the human hippocampal-entorhinal cortex. eLife. 2017;6:e17086. https://doi.org/ 10.7554/eLife.17086
39. Antonenko D, Diekelmann S, Olsen C, Born J, Molle M. Napping to renew learning capacity: enhanced encoding after stimulation of sleep slow oscillations. Eur J Neurosci. 2013;37:1142-52. https://doi.org/10.1111/ejn.12118

40. Grosmark AD, Mizuseki K, Pastalkova E, Diba K, Buzsaki G. REM sleep reorganizes hippocampal excitability. Neuron. 2012;75:1001-7. https://doi.org/10.1016/j. neuron.2012.08.015

41. Tononi G, Cirelli C. Sleep and the price of plasticity: from synaptic and cellular homeostasis to memory consolidation and integration. Neuron. 2014;81:12-34. https://doi.org/10.1016/j.neuron.2013.12.025

42. Frank MG. Erasing synapses in sleep: is it time to be SHY? Neural Plast. 2012;2012:264378 https://doi.org/10.1155/2012/264378

43. Hengen KB, Torrado Pacheco A, McGregor JN, Van Hooser SD, Turrigiano GG. Neuronal firing rate homeostasis is inhibited by sleep and promoted by wake. Cell. 2016;165:180-91. https://doi.org/10.1016/j. cell.2016.01.046

44. Hardt O, Nader K, Nadel L. Decay happens: the role of active forgetting in memory. Trends Cogn Sci. 2013;17:111-20. https://doi.org/10.1016/j. tics.2013.01.001

45. Migues PV, Liu L, Archbold GE, Einarsson EO, Wong J, Bonasia K, et al. Blocking synaptic removal of GluA2-containing AMPA receptors prevents the natural forgetting of long-term memories. J Neurosci. 2016;36:3481-94. https://doi.org/ 10.1523/JNEUROSCI.3333-15.2016

46. Villarreal DM, Do V, Haddad E, Derrick BE. NMDA receptor antagonists sustain LTP and spatial memory: active processes mediate LTP decay. Nat Neurosci. 2002;5:48-52. https://doi.org/10.1038/nn776

47. Beattie EC, Carroll RC, Yu X, Morishita W, Yasuda $H$, von Zastrow M, et al. Regulation of AMPA receptor endocytosis by a signaling mechanism shared with LTD. Nat Neurosci. 2000;3:1291-300. https://doi.org/10.1038/ 81823

48. Featherstone DE, Shippy SA. Regulation of synaptic transmission by ambient extracellular glutamate. Neuroscientist. 2008;14:171-81. https://doi.org/10.1177/ 1073858407308518

49. Ledgerwood L, Richardson R, Cranney J. Effects of D-cycloserine on extinction of conditioned freezing. Behav Neurosci. 2003;117:341-9.

50. Grillon C. D-cycloserine facilitation of fear extinction and exposure-based therapy might rely on lower-level, automatic mechanisms. Biol Psychiatry. 2009;66:636-41. https://doi.org/10.1016/j.biopsych.2009.04.017

51. Genzel L, Kiefer T, Renner L, Wehrle R, Kluge M, Grozinger M, et al. Sex and modulatory menstrual cycle effects on sleep related memory consolidation. Psychoneuroendocrinology. 2012;37:987-98. https://doi.org/10.1016/j. psyneuen.2011.11.006

52. Walker MP, Brakefield T, Seidman J, Morgan A, Hobson JA, Stickgold R, et al. Sleep and the time course of motor skill learning. Learn \& Mem. 2003;10:275-84. https://doi.org/10.1101//m.58503

53. Drosopoulos S, Schulze C, Fischer S, Born J. Sleep's function in the spontaneous recovery and consolidation of memories. J Exp Psychol General. 2007;136:169-83. https://doi.org/10.1037/0096-3445.136.2.169

54. Feld GB, Born J. Sculpting memory during sleep:concurrent consolidation and forgetting. Curr Opin Neurobiol. 2017;44:20-7. https://doi.org/10.1016/j. conb.2017.02.012 